\title{
Therapeutic Effect of Neuraminidase-1-Selective Inhibition in Mouse Models of Bleomycin-Induced Pulmonary Inflammation and Fibrosis
}

\author{
Irina G. Luzina, Erik P. Lillehoj, Virginia Lockatell, Sang W. Hyun, Katerina N. Lugkey, \\ Akihiro Imamura, Hideharu Ishida, Christopher W. Cairo, Sergei P. Atamas, ${ }^{1}$ \\ and Simeon E. Goldblum ${ }^{1}$ \\ Departments of Medicine (I.G.L., V.L., S.W.H., K.N.L., S.P.A., S.E.G.) and Pediatrics (E.P.L.), University of Maryland School of \\ Medicine, Baltimore, Maryland; Research Service, Baltimore VA Medical Center, Baltimore, Maryland (I.G.L., S.W.H., S.P.A., \\ S.E.G.); Department of Applied Bioorganic Chemistry, Gifu University, Gifu, Japan (A.I., H.I.); and Department of Chemistry, \\ University of Alberta, Edmonton, Alberta, Canada (C.W.C.)
}

Received July 16, 2020; accepted October 13, 2020

\begin{abstract}
Pulmonary fibrosis remains a serious biomedical problem with no cure and an urgent need for better therapies. Neuraminidases (NEUs), including NEU1, have been recently implicated in the mechanism of pulmonary fibrosis by us and others. We now have tested the ability of a broad-spectrum neuraminidase inhibitor, 2,3-dehydro-2-deoxy- $N$-acetylneuraminic acid (DANA), to modulate the in vivo response to acute intratracheal bleomycin challenge as an experimental model of pulmonary fibrosis. A marked alleviation of bleomycin-induced body weight loss and notable declines in accumulation of pulmonary lymphocytes and collagen deposition were observed. Real-time polymerase chain reaction analyses of human and mouse lung tissues and primary human lung fibroblast cultures were also performed. A predominant expression and pronounced elevation in the levels of NEU1 mRNA were observed in patients with idiopathic pulmonary fibrosis and bleomycin-challenged mice compared with their corresponding controls, whereas NEU2, NEU3, and NEU4 were expressed at far lower levels. The levels of mRNA for the NEU1 chaperone, protective protein/cathepsin A (PPCA), were also elevated by bleomycin. Western blotting analyses demonstrated bleomycin-induced elevations in protein expression of both NEU1 and PPCA in mouse lungs. Two known selective NEU1 inhibitors, C9-pentyl-amide-DANA
\end{abstract}

(C9-BA-DANA) and C5-hexanamido-C9-acetamido-DANA, dramatically reduced bleomycin-induced loss of body weight, accumulation of pulmonary lymphocytes, and deposition of collagen. Importantly, C9-BA-DANA was therapeutic in the chronic bleomycin exposure model with no toxic effects observed within the experimental timeframe. Moreover, in the acute bleomycin model, C9-BA-DANA attenuated NEU1mediated desialylation and shedding of the mucin-1 ectodomain. These data indicate that NEU1-selective inhibition offers a potential therapeutic intervention for pulmonary fibrotic diseases.

\section{SIGNIFICANCE STATEMENT}

Neuraminidase-1-selective therapeutic targeting in the acute and chronic bleomycin models of pulmonary fibrosis reverses pulmonary collagen deposition, accumulation of lymphocytes in the lungs, and the disease-associated loss of body weight-all without observable toxic effects. Such therapy is as efficacious as nonspecific inhibition of all neuraminidases in these models, thus indicating the central role of neuraminidase- 1 as well as offering a potential innovative, specifically targeted, and safe approach to treating human patients with a severe malady: pulmonary fibrosis.
This work was supported by National Institutes of Health National Heart, Lung, and Blood Institute [Grant R01-HL126897]; Department of Veterans Affairs [Grant I01CX000101] and [Grant I01BX002499]; and GlycoNet [Project CD-2]. We thank T. Guo (University of Alberta) for providing samples of CG33300. A PCT application relating to some of the findings described here has been filed with I.G.L., S.P.A., S.E.G., and C.W.C. as inventors. C.W.C. is a coinventor on patent filings related to CG33300.

${ }^{1}$ S.P.A. and S.E.G. equally contributing senior authors.

https://doi.org/10.1124/jpet.120.000223.

\section{Introduction}

Pulmonary fibrosis, a defining feature of interstitial lung disease, complicates a spectrum of illnesses (Spagnolo et al., 2018; Fischer and Distler, 2019; Perelas et al., 2020; Varone et al., 2020). It develops as a consequence of environmental or professional exposures (Khazdair et al., 2015; Trethewey and Walters, 2018; Greenberger, 2019; Hanania et al., 2019; Hoy and Chambers, 2020) or infections (Naik and Moore, 2010; George et al., 2020) or in its most severe forms emerges

ABBREVIATIONS: 4-MU-NANA, 2'-(4-methylumbelliferyl)- $\alpha$-D-N-acetylneuraminic acid; BAL, bronchoalveolar lavage; C9-BA-DANA, C9-pentylamide-DANA; cat., catalog number; CG33300, C5-hexanamido-C9-acetamido-DANA; COL, collagen; DANA, 2,3-dehydro-2-deoxy$\mathrm{N}$-acetylneuraminic acid; ED, ectodomain; IPF, idiopathic pulmonary fibrosis; KL-6, Krebs von den Lungen-6; MUC1, mucin-1; NEU, neuraminidase; PBS-T, PBS containing $0.05 \%$ Tween 20 ; PCR, polymerase chain reaction; PNA, peanut agglutinin; PPCA, protective protein/ cathepsin A; qRT-PCR, quantitative real-time polymerase chain reaction. 
idiopathically (Lederer and Martinez, 2018). The complex and redundant mechanisms of pulmonary fibrosis are not fully understood, and therapeutic options are limited. A labyrinthine network of cells and molecules contributing to pulmonary fibrosis has been identified, and new mediators continue to be added (Wynn, 2011; Todd et al., 2012; Luzina et al., 2015b; Glasser et al., 2016; Kolahian et al., 2016; Kurundkar and Thannickal, 2016). Each molecular mediator of pulmonary fibrosis represents a potential therapeutic target.

Numerous reports have associated elevated neuraminidase activity and expression with pulmonary fibrosis and mechanistically implicated neuraminidases (NEUs) (also called sialidases) (Lambré et al., 1988; Komleva et al., 1995; Luzina et al., 2016; Karhadkar et al., 2017, 2020). There are four known mammalian NEUs (NEU1, NEU2, NEU3, and NEU4) that belong to an ancient family of enzymes whose function is to remove terminal sialic acid (also known as neuraminic acid) from glycoconjugates. Of the four, NEU1 stands out as potentially relevant to fibrosis. NEU1 has been found to be irreplaceable in the physiologic formation of elastic fibers (Hinek et al., 2006; Duca et al., 2007), supporting the notion of its relevance to connective tissue biology. We recently reported that elevated NEU1 provokes profibrotic phenotypes in several pulmonary cell types, suggesting that therapeutic targeting of NEU1 may be efficacious in treating pulmonary fibrosis (Luzina et al., 2016).

An additional mechanistic and perhaps unexpected link between NEU1 and pulmonary fibrosis comes from previous studies of mucin-1 (MUC1) and its ectodomain (ED) (MUC1ED). Membrane-tethered MUC1 is comprised of a $>250 \mathrm{kDa}$ heavily sialylated MUC1-ED (Storr et al., 2008), a transmembrane domain, and a short cytoplasmic domain (Hattrup and Gendler, 2008; Lillehoj et al., 2013). The MUC1-ED also contains a Gly-Ser protease recognition site 58 amino acids upstream of the transmembrane domain, which upon desialylation is unmasked, permitting its proteolytic cleavage and release from the airway epithelial cell surface. The shed MUC1-ED containing the Krebs von den Lungen-6 (KL-6) epitope has been extensively used as a biomarker for pulmonary fibrotic disease activity (Yokoyama et al., 2006; Wakamatsu et al., 2017; Ishii et al., 2018; Raghu et al., 2018). Moreover, MUC1-ED has been implicated in pulmonary fibrosis mechanistically (Xu et al., 2013; Ballester et al., 2019, 2020; Milara et al., 2020). Anti-MUC1-ED antibody (Xu et al., 2013) and MUC1 gene deficiency (Milara et al., 2020) each protect against bleomycin-induced lung fibrosis in mice. We have identified MUC1 as an in vivo NEU1 substrate and demonstrated that MUC1-ED desialylation promotes its shedding (Lillehoj et al., 2015, 2019). Thus, the well-known increase in MUC1-ED levels in pulmonary fibrosis can be attributed to elevated NEU1 expression and catalytic activity. Inhibition of NEU1 catalytic activity may exert an antifibrotic effect by attenuating MUC1-ED desialylation and shedding.

The notion that mammalian NEUs may be targeted therapeutically has begun to be addressed in a proof-of-concept study (Karhadkar et al., 2017) wherein the authors demonstrated that oseltamivir (Tamiflu), a drug targeting the viral neuraminidase of the influenza virus, attenuated pulmonary fibrosis in the acute bleomycin mouse model. Although this is encouraging for the development of a neuraminidasetargeting approach, further experimental development of this concept is needed. Tamiflu was designed to target viral and not mammalian NEUs. It lacks NEU1 inhibitory activity at concentrations up to $1 \mathrm{mM}$. Its $\mathrm{IC}_{50}$ for NEU1 is rather high at $\sim 50 \mathrm{mM}$, and its inhibitory activity for other mammalian NEUs is essentially undetectable (Hata et al., 2008; Lindemann et al., 2010; Richards et al., 2018). Oseltamivir is approved for short-term treatment and prophylaxis of influenza but is not recommended long-term because of its notable side effects (Hata et al., 2008; Gupta et al., 2015; Heneghan et al., 2016). We previously established elevated NEU1 expression in the lung tissues of patients with idiopathic pulmonary fibrosis (IPF) (Luzina et al., 2016). These combined considerations prompted our exploration of the potential for pharmacological inhibitors that target mammalian NEU1. Two recently described noncovalent competitive inhibitors that selectively target NEU1, C9-pentyl-amide-DANA (C9-BA-DANA) (Magesh et al., 2008; Hyun et al., 2016; Lillehoj et al., 2019), and C5-hexanamido-C9-acetamidoDANA (CG33300) (Guo et al., 2018; Howlader et al., 2020) have been tested for the first time in this study for their ability to mitigate pulmonary fibrosis in vivo.

\section{Materials and Methods}

Neuraminidase Inhibitors. Pan-neuraminidase inhibitor 2,3dehydro-2-deoxy- $N$-acetylneuraminic acid (DANA) was purchased from Calbiochem (La Jolla, CA). NEU1-selective inhibitor C9BA-DANA, referred to as " $10 \mathrm{~h}$ " in the original publication, was synthesized as described (Magesh et al., 2008). An alternative NEU1selective inhibitor, C5-hexanamido-C9-acetamido-DANA, referred to as " $17 \mathrm{f}$ " in the original publication and as "CG33300" here, was synthesized as described (Guo et al., 2018) and used here in its methyl ester form CG33301.

Animal Studies. Wild-type female C57BL/6 mice aged 1012 weeks and weighing 18-20 g (The Jackson Laboratory, Bar Harbor, $\mathrm{ME}$ ) were treated in accordance with a research protocol approved by the University of Maryland Institutional Animal Care and Use Committee. Animals were maintained in sterile microisolator cages with sterile rodent feed and water in the Baltimore VA Medical Center Research Animal Facility as approved by the Association for Assessment and Accreditation of Laboratory Animal Care.

In Vivo Inhibition of Neuraminidase Activity. Mice were injected intraperitoneally with $15 \mu \mathrm{g} / \mathrm{kg}$ of DANA, C9-BA-DANA, CG33300, or PBS control intraperitoneally. After 24 hours, mice were injected intraperitoneally with a second dose of the same inhibitor or PBS control. After an additional 18 hours, the mice were euthanized; lungs were harvested, weighed, homogenized, and centrifuged; and the supernatants were processed for total neuraminidase activity for the fluorogenic substrate, 2'-(4-methylumbelliferyl)- $\alpha$-D- $N$-acetylneuraminic acid (4-MU-NANA) as described (Cross et al., 2012; Hyun et al., 2016).

Acute Bleomycin Lung Injury Model. To induce pulmonary inflammation and fibrosis, a single dose of $0.075 \mathrm{U}$ of bleomycin (Sigma-Aldrich, St. Louis, MO) diluted in $50 \mu$ l of sterile PBS was delivered intratracheally to mouse lungs on day 0 as described (Pochetuhen et al., 2007; Luzina et al., 2013, 2015a). Briefly, a minor anterior midline neck incision was made to reveal the trachea, a Micro Sprayer (Penn-Century, Wyndmoor, Philadelphia, PA) was inserted intratracheally, and bleomycin or sterile PBS was instilled. Mice were serially weighed, and on days 8-13, they were daily administered intraperitoneally with DANA, C9-BA-DANA, and CG33300, all dissolved in PBS, in doses indicated below in each of the experiments, or PBS control. On days 14 or 21 , mice were euthanized by $\mathrm{CO}_{2}$ asphyxiation followed by cervical dislocation. Immediately postmortem, bronchoalveolar lavage (BAL) samples and lungs were harvested. BAL samples were collected and analyzed as described (Pochetuhen et al., 2007; Luzina et al., 2013, 2015a). Briefly, installation and withdrawal 
of $1 \mathrm{ml}$ of PBS twice via an 18-gauge blunt-end needle secured in the trachea were performed in each animal. The two aliquots of BAL were pooled and centrifuged, and total and differential cell counts were performed. The lungs were processed for quantitative real-time polymerase chain reaction (qRT-PCR), quantitative immunoblotting, and determination of hydroxyproline as a measure of total collagen as previously described (Pochetuhen et al., 2007; Luzina et al., 2013, 2015a).

Chronic Bleomycin Injury Model. Repeated intraperitoneal injections of bleomycin were used in the chronic injury model as described (Baran et al., 2007; Zhou et al., 2011; Luo et al., 2016). Briefly, $0.018 \mathrm{U} / \mathrm{g}$ of bleomycin or PBS control was injected twice a week for 33 days, whereas C9-BA-DANA or PBS control was injected intraperitoneally twice a week starting on day 14 . The lungs were processed for total collagen.

Quantitative Reverse Transcriptase-Polymerase Chain Reactions. Total cellular RNA was extracted from murine lung tissue, as described using Trizol (ThermoFisher Scientific, Waltham, MA), and RNA purity was established based on the $A_{260} / A_{280}$ absorption ratio. Total RNA $(1.0 \mu \mathrm{g})$ was treated with DNase I (Invitrogen ThermoFisher) for 15 minutes and reverse-transcribed using avian myeloblastosis virus reverse transcriptase and poly(T) primer (Promega, Madison, WI). The resulting cDNA was quantified by qRT-PCR using prevalidated primers for COL1A2, COL3A1, NEU1, NEU2, NEU3, NEU4, and PPCA as well as RT2 SYBR Green qPCR Master Mix, all of which were from Qiagen (Germantown, MD). For thermal cycling, a StepOnePlus Real-Time PCR System (ThermoFisher) was used.

Immunoblotting. Murine lung homogenates were mixed with equal volumes of Laemmli loading buffer supplemented with $\beta$-mercaptoethanol and boiled and resolved by SDS-PAGE. After wet electrotransfer to polyvinylidene difluoride membranes (PVDF), the membranes were blocked and incubated with primary and secondary antibody using Tris-buffered saline with $0.1 \%$ Tween 20 and $5 \%$ bovine serum albumin. The following primary antibodies were used: rabbit anti-human NEU1 (cat. TA335236; OriGene, Rockville, MD) and rabbit anti-human NEU2, NEU3, and NEU4, which were also from OriGene; rabbit anti-human PPCA (cat. ab184553; Abcam, Cambridge, MA); and rabbit anti-human glyceraldehyde-3-phosphate dehydrogenase from Cell Signaling Technology (cat. 5174; Dancers, MA). Horseradish peroxidase-tagged secondary antibody was goat antirabbit IgG from EMD Millipore (cat. 12-348; Billerica, MA). Chemiluminescent signal was developed using SuperSignal West Pico Chemiluminescent Substrate (ThermoFisher). Membrane stripping was performed using ReBlot Plus stripping solution (EMD Millipore).

For determination of desialylated MUC1-ED, equal protein aliquots of bronchioalveolar lavage fluid were incubated with peanut agglutinin (PNA) immobilized on agarose beads to enrich for PNA-binding as described (Lillehoj et al., 2019). The PNA-binding proteins were resolved by SDS-PAGE and transferred to PVDF membrane. The blots were developed with anti-MUC1-ED antibody (cat. LS-C343984; LifeSpan Biosciences, Seattle, WA) followed by horseradish peroxidase-conjugated secondary antibody.

Measurement of Total Lung Collagen. Total lung collagen was measured based on the quantification of hydroxyproline using the colorimetric QuickZyme assay (QuickZyme BioSciences, Leiden, The Netherlands) according to the manufacturer's recommendations. Briefly, after hydrolysis of $50 \mathrm{mg}$ lung tissue in $500 \mu \mathrm{l}$ of $6 \mathrm{M} \mathrm{HCl}$ for 20 hours at $95^{\circ} \mathrm{C}$, the hydrolysate was diluted 10 -fold with $4 \mathrm{M} \mathrm{HCl}$ and measured against serial dilutions of collagen standard. The results were expressed as microgram collagen per milligram wet lung tissue.

ELISA for the Murine MUC1- ED. Murine bronchoalveolar lavage supernatants were centrifuged at $10,000 \mathrm{~g}$ for 10 minutes at $4^{\circ} \mathrm{C}$, transferred in triplicates to 96 -well Nunc MaxiSorp plates (ThermoFisher), and incubated overnight at $4^{\circ} \mathrm{C}$. Wells were blocked for 1 hour at room temperature with PBS, $\mathrm{pH} 7.0$, containing $10 \mathrm{mg} / \mathrm{ml}$ bovine serum albumin and $50 \mathrm{mg} / \mathrm{ml}$ sucrose and washed with PBS containing $0.05 \%$ Tween 20 (PBS-T). The samples were reacted for 2 hours at room temperature with $200 \mu \mathrm{g} / \mathrm{ml}$ rabbit anti-murine
MUC1-ED antibody (cat. LS-C343984; LifeSpan Biosciences, Seattle, WA), washed with PBS-T, reacted for 2 hours at room temperature with $200 \mu \mathrm{g} / \mathrm{ml}$ of horseradish peroxidase-conjugated goat anti-rabbit IgG secondary antibody, and washed with PBS-T. Bound antibodies were detected with tetramethylbenzidine substrate. The substrate reaction was stopped with $1 \mathrm{~N} \mathrm{HCl}$, and $A_{450}$ was measured. A standard curve using purified murine MUC1-ED was generated for each ELISA plate as described (Lillehoj et al., 2019).

Statistical Analysis. Experimental data were expressed as mean + S.D. values. Pairwise comparisons of groups were performed utilizing two-tailed Student's $t$ test. Multiple groups were analyzed using one-way ANOVA.

\section{Results}

Effect of Pan-Neuraminidase Inhibition in the Acute Bleomycin Lung Injury Model. We previously reported that a noncovalent competitive pan-neuraminidase inhibitor, DANA, inhibited total neuraminidase activity in human airway epithelia and lung microvascular endothelia (Cross et al., 2012; Lillehoj et al., 2012). Our previous study estimated an $\mathrm{IC}_{50}$ of a DANA derivative, C9-BA-DANA, for total neuraminidase activity in vitro as well as demonstrated that both DANA and its NEU1-selective derivative C9-BA-DANA potently inhibit neuraminidase activity in mouse lungs in vivo at $15 \mu \mathrm{g} / \mathrm{kg}$ (Hyun et al., 2016). We now have further corroborated the ability of DANA to attenuate neuraminidase activity in mouse lungs in vivo. Two $15 \mu \mathrm{g} / \mathrm{kg}$ doses of DANA were administered intraperitoneally at 42 hours and 18 hours prior to harvesting lungs, causing a reduction in total neuraminidase activity for the 4-MU-NANA substrate by 99.2\% (Fig. 1A).

Since previous studies suggest neuraminidase involvement in pulmonary fibrosis (Joseph et al., 1989; Luzina et al., 2016; Karhadkar et al., 2020), we tested the effect of this panneuraminidase inhibitor in the acute bleomycin injury model. These tests were performed in the therapeutic but not preventive mode, considering that human patients with pulmonary fibrotic diseases are commonly diagnosed after the fibrotic process in the lungs is already established. Mice were challenged with a single intratracheal instillation of bleomycin, and the disease was allowed to evolve over 7 days. To choose a dosing regimen of DANA, we relied on existing reports (Chairat et al., 2013; Hyun et al., 2016). Anti-influenza neuraminidase inhibitor, all derivatives of DANA were tested in humans and showed elimination half-lives varying among the tested compounds. Oseltamivir had a half-life of approximately 7.7 hours after oral administration, whereas half-life of injected peramivir varied between 7.7 and 20.8 hours (Chairat et al., 2013). We therefore considered a daily administration of DANA to be suitable for our original study of DANA in pulmonary fibrosis in vivo. On days 7-14, mice were injected daily intraperitoneally with $15 \mu \mathrm{g} / \mathrm{kg}$ of DANA or PBS vehicle. The treatment with DANA elicited a rapid reversal of the bleomycin-induced loss of body weight (Fig. 1B). Total and differential cell counts in bronchoalveolar lavage samples collected on day 14 after bleomycin injury (day 7 of treatment) revealed that pan-neuraminidase inhibition with DANA attenuated bleomycin-induced accumulation of total cells and, specifically, lymphocytes (Fig. 1C). qRT-PCR analyses of lung homogenates revealed that the steady-state levels of mRNAs for collagen chains COL1A2 and COL3A1 were elevated in bleomycin-challenged mice but attenuated in mice 
A

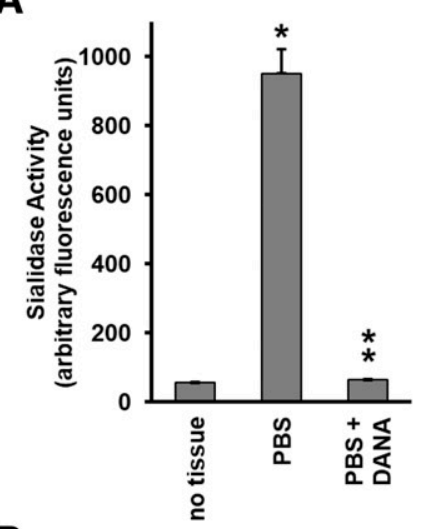

D

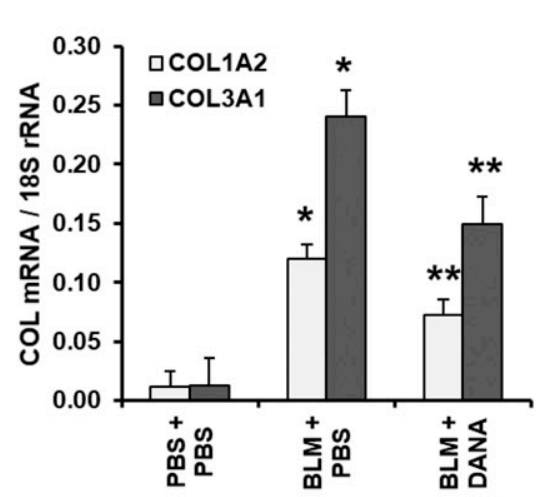

B

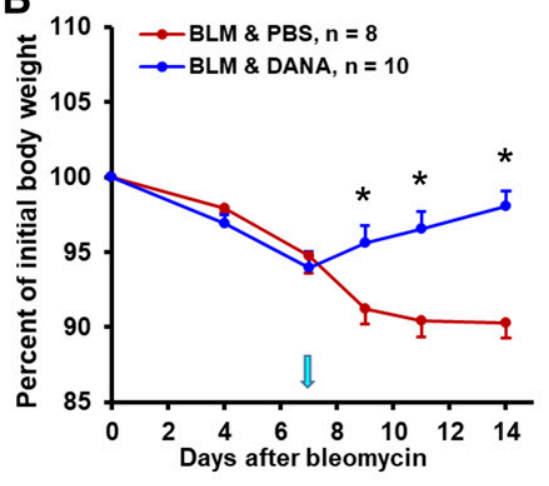

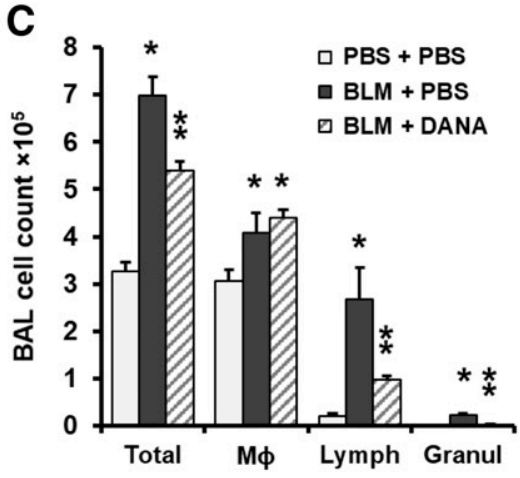

E

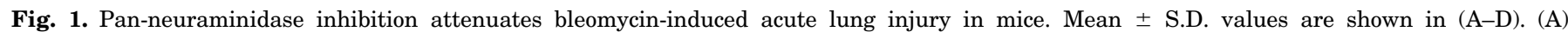

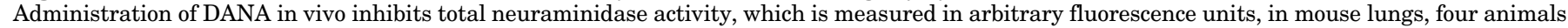

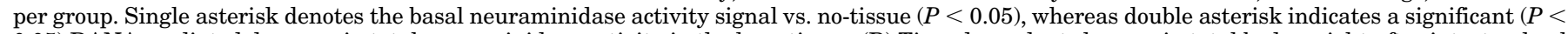

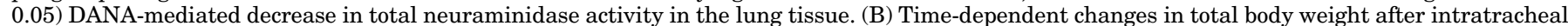

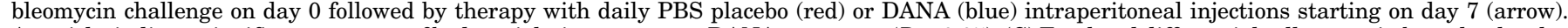

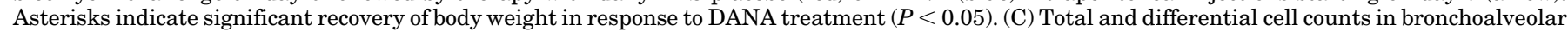

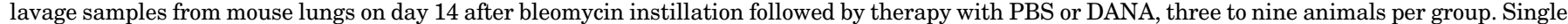

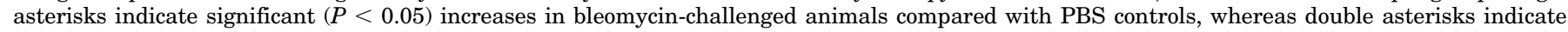

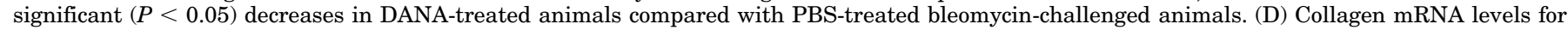

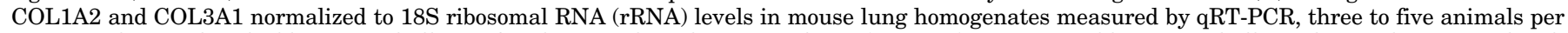

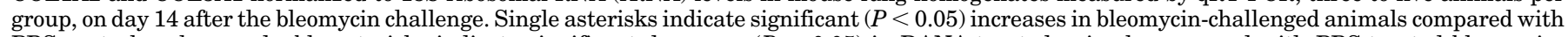

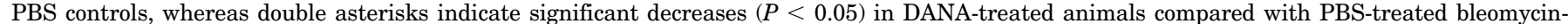

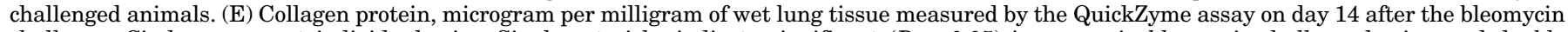

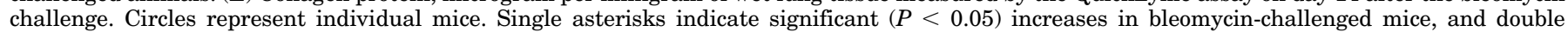

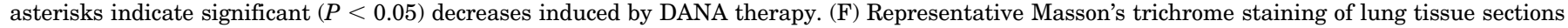
from mice challenged with intratracheal PBS or bleomycin and treated with PBS or DANA as indicated on day 14.

that received DANA (Fig. 1D). Furthermore, at the protein level, bleomycin injury increased pulmonary levels of collagen, whereas subsequent treatment with DANA attenuated such increases (Fig. 1E). Histologically, treatment with DANA protected against inflammatory infiltration and collagen deposition (Fig. 1F). These combined data indicate that panneuraminidase inhibition in the acute bleomycin injury model restores total body weight, limits pulmonary lymphocytic infiltration and collagen accumulation, and decreases histopathological changes in the lungs.

Relative Expression of Neuraminidases in Lung Tissue and Cultured Lung Fibroblasts. The observed protective therapeutic effect of pan-neuraminidase inhibition on bleomycin-induced injury led us to ask whether one or more of the four known mammalian neuraminidases (NEU1-NEU4) may be at play. Deidentified lung tissues that previously banked in separate institutional review board-approved studies from human patients with IPF and adult healthy controls were analyzed by qRT-PCR for NEU1, NEU2, NEU3, and
NEU4 expression using specific primers. The steady-state mRNA levels of all four neuraminidases were elevated in patients with IPF, with average fold increases from healthy control tissues of 2.6-fold for NEU1, 37.2-fold for NEU2, 10.7fold for NEU3, and 47.9-fold for NEU4 (Fig. 2A). Such foldchange increases alone are somewhat misleading considering that NEU2, NEU3, and NEU4 mRNAs are present in low levels in lung tissues from healthy controls and that NEU1 mRNA levels are predominant over other NEUs in lung tissues from healthy controls and patients with IPF (Fig. 2A). Similar analyses of primary lung fibroblast cultures from human patients with IPF and healthy controls revealed a statistically significant $(P=0.043)$ but modest 1.2 -fold elevation in NEU1 but not the other three neuraminidases (Fig. 2B). These findings suggested that although all four neuraminidases may be involved in the disease process, the relative contribution from NEU1 may be greater than that from NEU2-NEU4. In the acute bleomycin injury model, NEU1 mRNA was most pronouncedly elevated in response to 
A

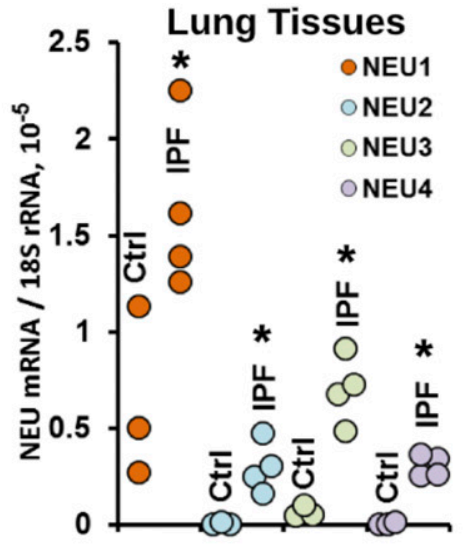

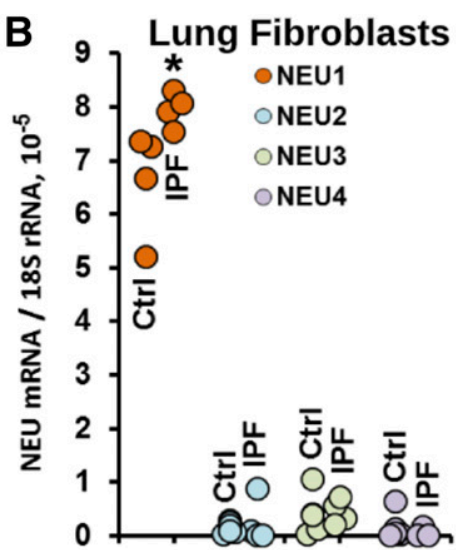

C

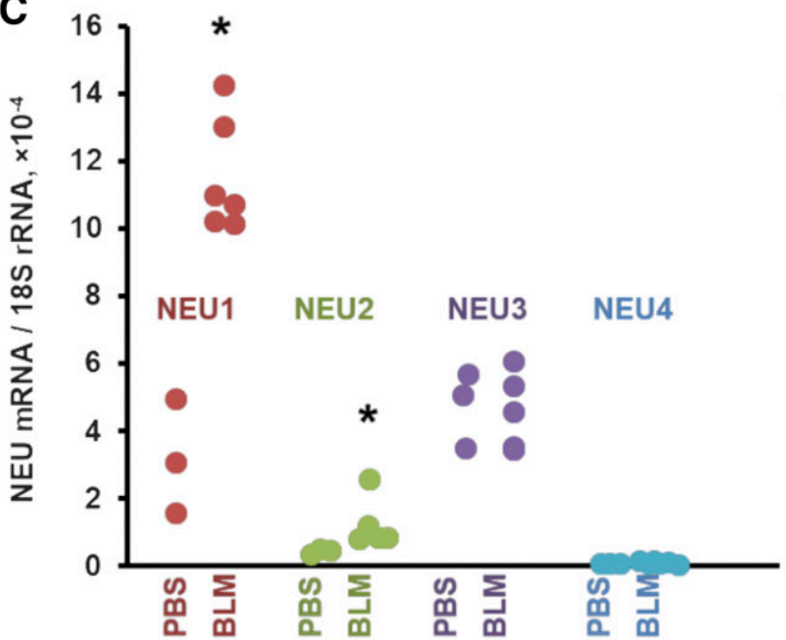

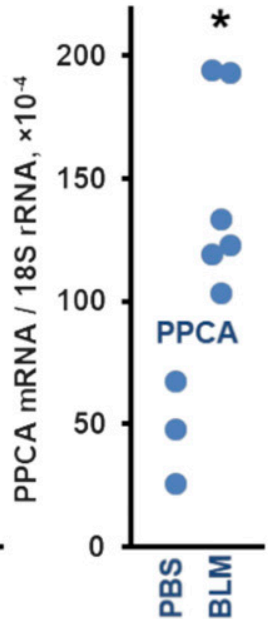

Fig. 2. Neuraminidase and PPCA expression in lung tissues and fibroblasts. Human lung tissues (A) and primary lung fibroblasts (B) from healthy controls (Ctrl) and patients with IPF were analyzed using qRT-PCR for indicated neuraminidases. Each symbol indicates a separate donor of lung tissues or fibroblasts. Lung tissues from PBS-challenged control and bleomycin-challenged mice were also analyzed for neuraminidase and PPCA mRNA levels $(\mathrm{C})$, with each symbol indicating a separate mouse. In $(\mathrm{A}-\mathrm{C})$, asterisks indicate significant $(P<0.05)$ differences from the corresponding controls. Immunoblotting assays of lung homogenates revealed elevations in NEU1 and PPCA protein levels in bleomycin-challenged mice (D); glyceraldehyde-3-phosphate dehydrogenase (GAPDH)-normalized band optical densities (OD) are indicated below corresponding lanes. rRNA, ribosomal RNA.

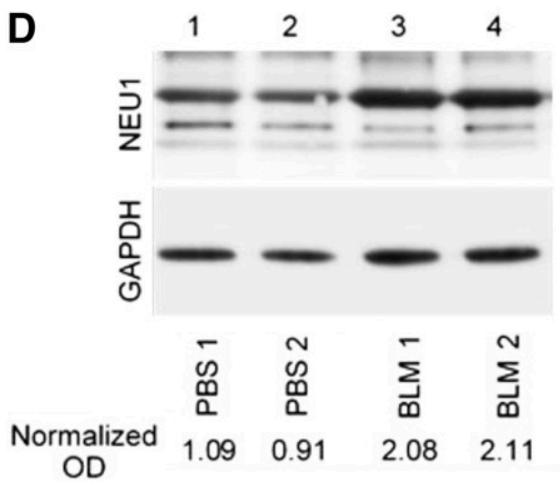

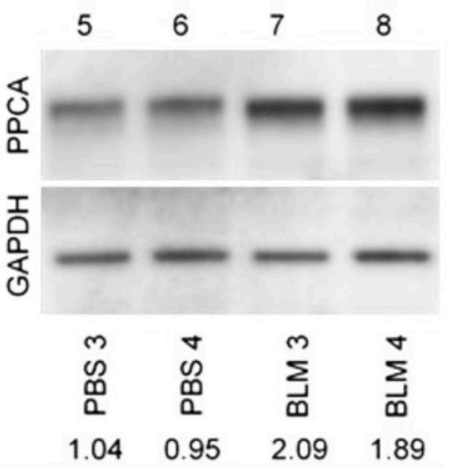

the injury compared with mRNAs for the other three NEUs (Fig. 2C). The levels of mRNA for the NEU1 chaperone, PPCA, were also elevated in bleomycin-challenged animals (Fig. 2C). The levels of NEU1 and PPCA proteins were similarly elevated in mice treated with bleomycin (Fig. 2D), whereas other NEUs were not detectable by Western blotting in lung tissues of either PBS- or bleomycin-treated mice. These observations suggest a predominant role for NEU1 in pulmonary fibrotic injury, although contributions from other neuraminidases cannot be excluded.

Effect of NEU1-Selective Inhibition with C9-BA-DANA in the Acute Bleomycin Injury Model. Unlike DANA, which is a competitive inhibitor of all mammalian NEUs, C9BA-DANA and CG33300 are selective inhibitors of NEU1 with
$\mathrm{IC}_{50}$ values of 3 and $0.05 \mu \mathrm{M}$, respectively, and selectivity over other NEUs of 35 -fold or higher and 340 -fold or higher, respectively (Magesh et al., 2008; Guo et al., 2018; Howlader et al., 2020). To assess the relevant contribution of NEU1 to pulmonary fibrotic injury in the acute bleomycin model, NEU1-selective inhibition with C9-BA-DANA (Magesh et al., 2008; Hyun et al., 2016; Lillehoj et al., 2019) was employed. We previously reported that C9-BA-DANA inhibits NEU1 catalytic activity in cultured human pulmonary epithelial and endothelial cells as well as fibroblasts (Hyun et al., 2016). Furthermore, C9-BA-DANA inhibited NEU1 catalytic activity in mice in vivo (Hyun et al., 2016; Lillehoj et al., 2019). To validate those initial observations, two doses of $15 \mu \mathrm{g} / \mathrm{kg}$ C9-BA-DANA were administered intraperitoneally 24 hours 
apart, and total lung neuraminidase activity was measured. Such treatment reduced total lung neuraminidase activity by 99.8\% (Fig. 3A). This observation further validates the notion that most of the total neuraminidase activity in the lung can be ascribed to NEU1. Similar to the effects of panneuraminidase inhibition (Fig. 1), NEU1-selective therapy with C9-BA-DANA reversed the bleomycin-induced loss of total body weight (Fig. 3B) and attenuated pulmonary infiltration of lymphocytes (Fig. 3C) and collagen protein deposition (Fig. 3D) as well as diminished bleomycin injuryinduced histopathological changes (Fig. 3E). These findings indicate that NEU1-selective inhibition is therapeutic in the acute bleomycin model of pulmonary inflammation and fibrosis.

Long-Term Effect of NEU1-Selective Inhibition with C9-BA-DANA on Bleomycin-Induced Injury. In the acute bleomycin injury model, inflammation peaks on days 7-14 and partially resolves by day 21, whereas fibrotic deposits are clearly observable on days 14 through 21 (Degryse and Lawson, 2011; Moore et al., 2013; Carrington et al., 2018; O'Dwyer and Moore, 2018). Additional experiments were performed to assess the effects of therapy with C9-BA-DANA up to 21 days in the acute bleomycin injury model. Furthermore, based on available data on dose-dependent NEU1 inhibition by C9-BA-DANA (Hyun et al., 2016), it was considered a possibility that lower doses of this inhibitor might have a therapeutic effect. Daily intraperitoneal administration of 1,5 , or $15 \mu \mathrm{g} / \mathrm{kg}$ of C9-BA-DANA starting on day 7 after intratracheal instillation of bleomycin dose-dependently reversed bleomycin-induced loss of total body weight (Fig. 4A). The levels of lung collagen were decreased in bleomycinchallenged mice by subsequent administration of either 1, 5, or $15 \mu \mathrm{g} / \mathrm{kg}$ of C9-BA-DANA not only after 7 days of treatment (day 14 after bleomycin challenge, Fig. 3D; Fig. 4B) but also after 14 days of treatment ( 21 days after bleomycin challenge, Fig. 4C). These findings indicate that the lower dose of $5 \mu \mathrm{g} / \mathrm{kg}$ of C9-BA-DANA is comparable to its therapeutic effect at the higher $15 \mu \mathrm{g} / \mathrm{kg}$ dose of the drug and that the effect is
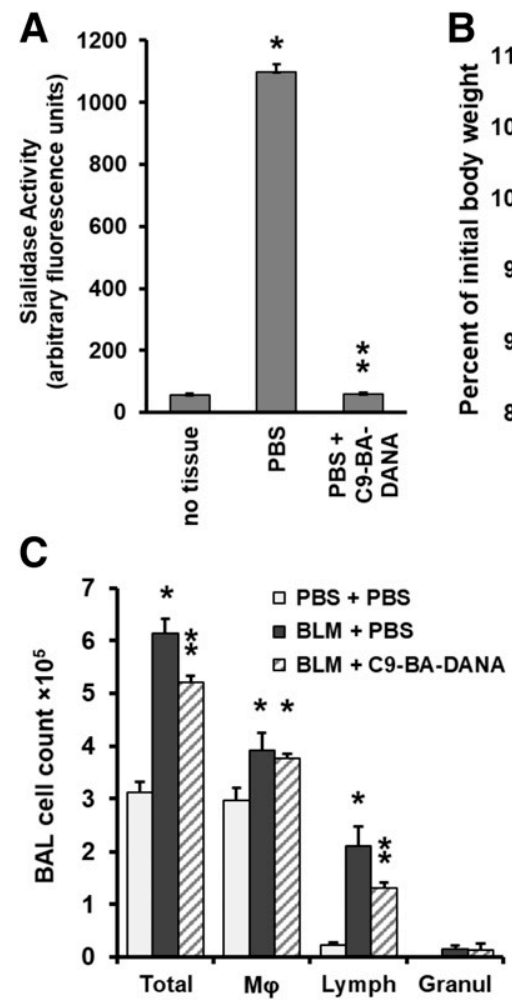

B
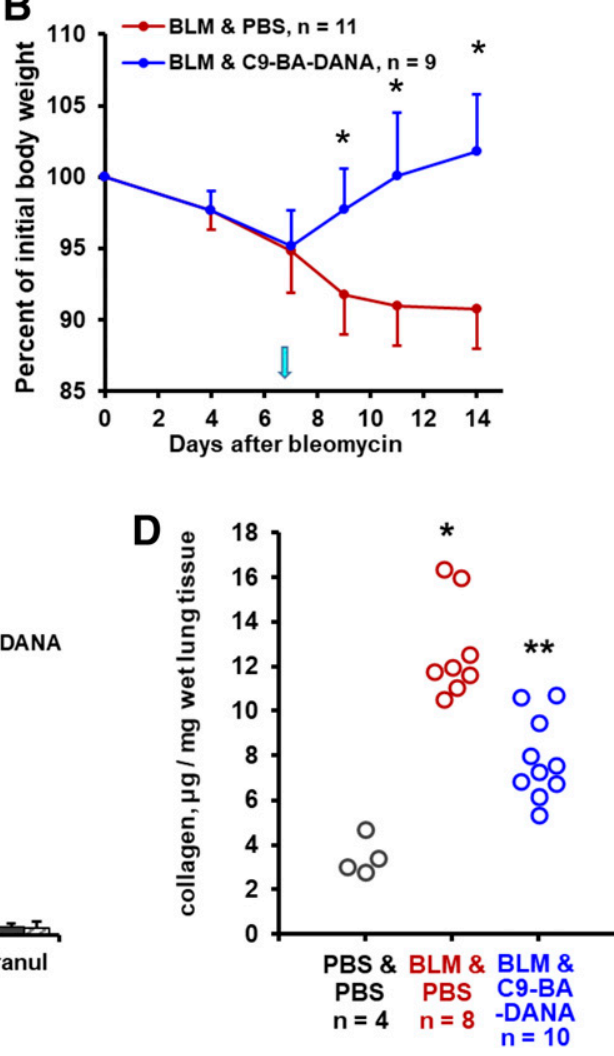

E

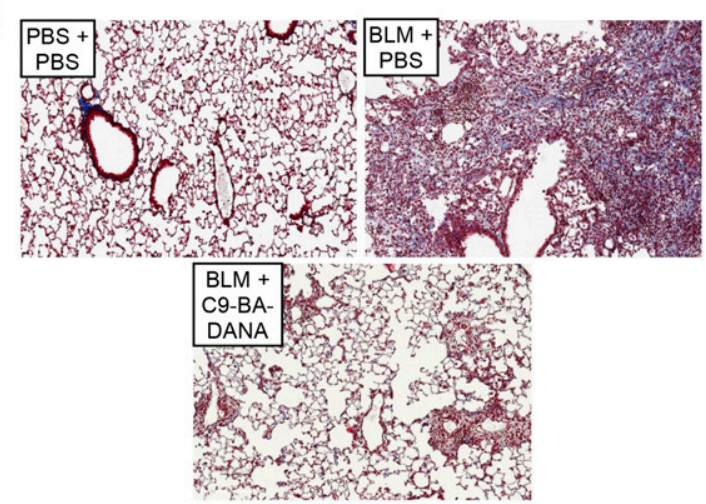

Fig. 3. NEU1-selective inhibition with C9-BA-DANA attenuates bleomycin-induced acute lung injury in mice. Mean \pm S.D. values are shown in (A-C). (A) Administration of C9-BA-DANA in vivo inhibits total neuraminidase activity, which is measured in arbitrary fluorescence units, in mouse lungs, four animals per group. Single asterisk denotes the basal total neuraminidase activity signal vs. no tissue $(P<0.05)$, whereas double asterisk indicates a significant $(P<0.05)$ DANA-mediated decrease in total neuraminidase activity in lung tissue. (B) Time-dependent changes in total body weight after intratracheal bleomycin challenge on day 0 followed by therapy with daily PBS placebo (red) or C9-BA-DANA (blue) intraperitoneal injections starting on day 7 (arrow). Asterisks indicate significant $(P<0.05)$ recovery of body weight in response to $C 9$ BA-DANA treatment. (C) Total and differential cell counts in bronchoalveolar lavage samples from mouse lungs on day 14 after bleomycin instillation followed by therapy with PBS or C9-BA-DANA, 4 to 10 animals per group. Single asterisks indicate significant $(P<0.05)$ increases in bleomycin-challenged animals compared with PBS controls, whereas double asterisks indicate significant decreases $(P<0.05)$ in C9-BA-DANA-treated compared with PBS-treated bleomycin-challenged animals. (D) Collagen protein, microgram per milligram of wet lung tissue measured based on hydroxyproline assay (QuickZyme) on day 14 after the bleomycin challenge. Circles represent individual mice. Single asterisks indicate significant $(P<$ 0.05 ) increases in bleomycin-challenged mice, and double asterisks indicate significant $(P<0.05)$ decreases induced by C9-BA-DANA therapy. (E) Representative Masson's trichrome staining of lung tissue sections from mice challenged with intratracheal PBS or bleomycin and treated with PBS or C9-BA-DANA as indicated on day 14. 
A

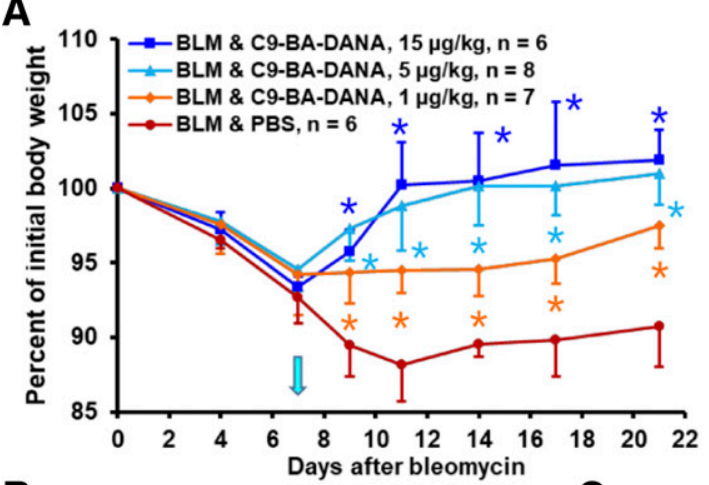

B

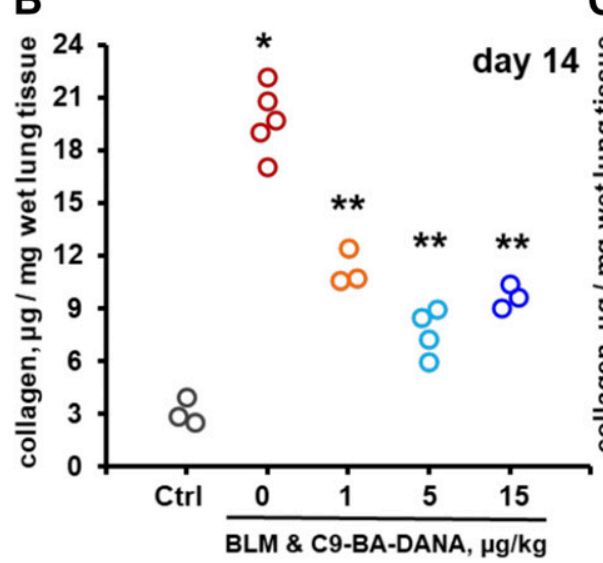

\section{D}

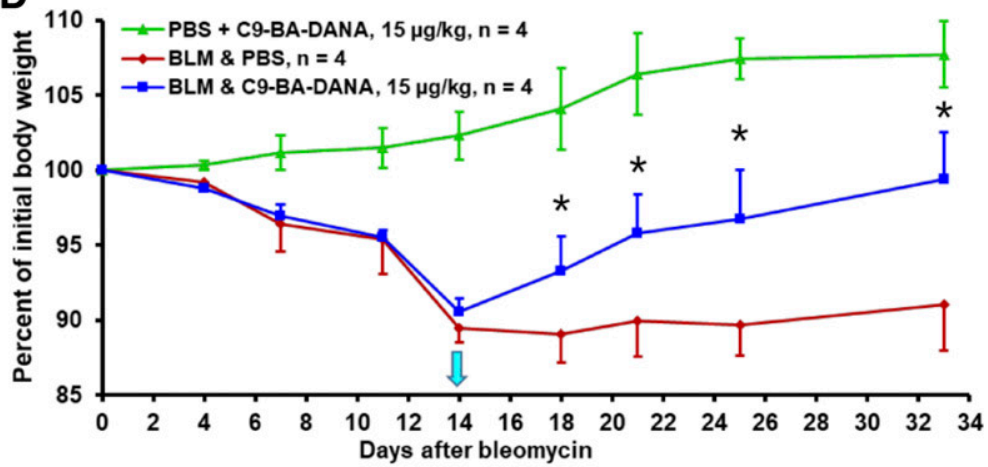

$\mathrm{E}$

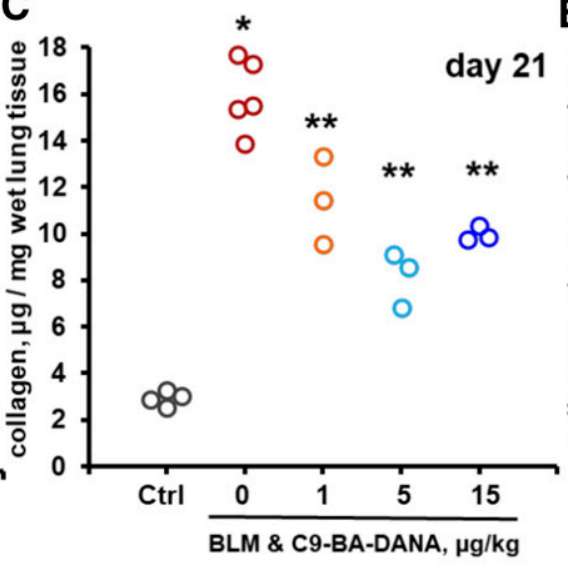

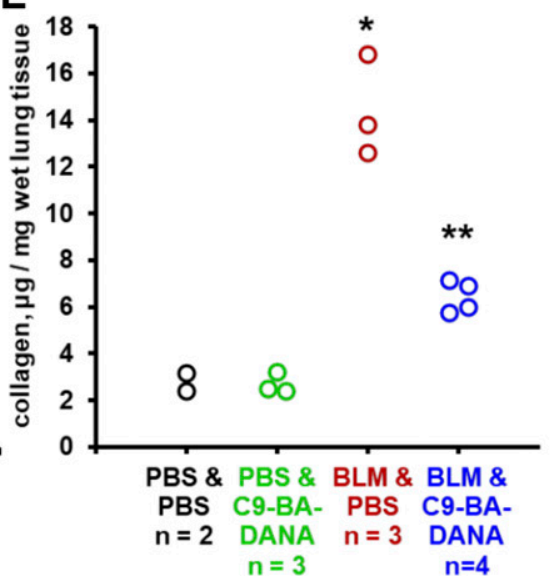

Fig. 4. NEU1-selective inhibition with C9-BA-DANA has a long-lasting therapeutic effect. (A-C) show findings in the acute injury model up to day 21 after bleomycin instillation, whereas (D and E) show findings in the chronic bleomycin challenge model. Mean \pm S.D. values are shown in (A and D), whereas circles represent individual mice in $(\mathrm{B}, \mathrm{C}$, and $\mathrm{E})$. In $(\mathrm{A}$ and $\mathrm{D})$, asterisks indicate significant $(P<0.05)$ recovery of body weight in response to C9-BA-DANA treatment. In (B, C, and E), single asterisks indicate significant $(P<0.05)$ increases in collagen levels in bleomycin-challenged mice, and double asterisks indicate significant $(P<0.05)$ decreases induced by C9-BA-DANA therapy. (A) Time-dependent changes in total body weight after intratracheal bleomycin challenge on day 0 followed by therapy with daily intraperitoneal injections of PBS placebo (red) or indicated doses of C9-BADANA starting on day 7 (arrow). (B) Collagen protein in microgram per milligram of wet lung tissue measured based on hydroxyproline assay (QuickZyme) on day 14 after the bleomycin challenge with and without treatment with indicated doses of C9-BA-DANA. (C) Collagen protein in microgram per milligram of wet lung tissue measured based on hydroxyproline assay (QuickZyme) on day 21 after the bleomycin challenge with and without treatment indicated doses of C9-BA-DANA. (D) Time-dependent changes in total body weight in mice challenged with repeated intraperitoneal injections of bleomycin throughout the study period. Therapy with PBS placebo (red) or C9-BA-DANA (blue) was started on day 14 (arrow). Mice infused with PBS alone and treated with C9-BA-DANA are shown in green. (E) Collagen protein in microgram per milligram of wet lung tissue measured with the hydroxyproline assay (QuickZyme) on day 33, with and without treatment with C9-BA-DANA. Ctrl, control.

long-lasting. The therapeutic effect was less pronounced yet significant and persistent with $1 \mu \mathrm{g} / \mathrm{kg}$ of C9-BA-DANA (Fig. 4, A-C).

Despite the broad use of the acute bleomycin injury model, it has several limitations, which somewhat diminish its relevance to human pulmonary fibrosis. In contrast to this model, human pulmonary fibrotic diseases are chronic in nature, have a characteristic histopathological appearance that is not fully reproduced by the acute bleomycin model, and have a notably less pronounced inflammatory component than that observed in mice acutely challenged with bleomycin (Degryse and Lawson, 2011; Moore et al., 2013; Carrington et al., 2018; O'Dwyer and Moore, 2018). To address these limitations, a chronic bleomycin exposure model was developed in which pulmonary fibrosis was accompanied by modest inflammation as well as histopathological characteristics resembling those of human pulmonary fibrotic pathology. In this model, bleomycin was delivered systemically, either with a subcutaneously placed osmotic pump (Lee et al., 2014b; Ravanetti et al., 2020) or by repetitive systemic injections (Zhou et al., 2011; Weng et al., 2019). We used the latter model by administering bleomycin intraperitoneally twice a week for 4 weeks. The treatment with $15 \mu \mathrm{g} / \mathrm{kg}$ of C9-BA-DANA administered twice a week (alternating with bleomycin administrations) was initiated on day 14. Mice were followed up until day 33 after initiation of chronic bleomycin administration. In the chronic bleomycin injury model, therapy with C9-BA-DANA attenuated the loss of body weight (Fig. 4D) and accumulation of collagen in the lungs (Fig. 4E).

Along with assessment of therapeutic efficacy, such prolonged treatment offers an opportunity for a detailed evaluation of possible toxic effects of C9-BA-DANA. A group of mice were administered PBS instead of bleomycin and received an equivalent dosing regimen of C9-BA-DANA. In this C9-BADANA-exposed group without underlying bleomycin-driven disease, no changes in food or water consumption or grooming behavior were observed. The C9-BA-DANA therapy alone did not cause ataxia, hunched posture, or ruffled fur in any of the animals at any of the time points. Mice were gaining weight as expected for their age (Fig. 4D). Postmortem histologic assessment of spleen, liver, kidney, or heart did not reveal observable differences from PBS-treated control mice. The 
treatment with C9-BA-DANA did not affect the basal levels of pulmonary collagen (Fig. 4E). Therefore, C9-BA-DANA did not exert detectable toxicity within the experimental time frame.

NEU1-Selective Inhibition Attenuates MUC-1 Ectodomain Desialylation and Shedding. We had previously identified MUC1 as an in vivo NEU1 substrate (Lillehoj et al., 2012). We also found that NEU1-mediated MUC1-ED desialylation plays a permissive role for its proteolytic cleavage and shedding both in vitro (Lillehoj et al., 2015) and in vivo (Lillehoj et al., 2019). It is therefore conceivable that the observed involvement of NEU1 in the bleomycin model of pulmonary fibrosis might be accompanied by MUC1-ED desialylation and elevated shedding and that NEU1selective inhibition might prevent this process. Indeed, MUC1-ED was elevated in bronchoalveolar lavage samples from bleomycin-challenged mice, and C9-BA-DANA inhibited its release in a dose-dependent fashion (Fig. 5A). The increased NEU1 expression and NEU1-mediated MUC1-ED desialylation and shedding may well explain the utility of KL6 as a biomarker for IPF disease activity (Yokoyama et al., 2006; Wakamatsu et al., 2017; Ishii et al., 2018; Raghu et al., 2018) and further support the notion of its mechanistic involvement in the disease process (Xu et al., 2013; Ballester et al., 2019, 2020; Milara et al., 2020). We simultaneously assessed the sialylation state of shed MUC1-ED in bronchoalveolar lavage fluid samples using PNA lectin to selectively recognize desialylated MUC1-ED. Consistent with our previous findings in other models (Lillehoj et al., 2012, 2015, 2019), shed MUC1-ED was desialylated in bleomycinchallenged mice, and C9-BA-DANA inhibited MUC1-ED desialylation (Fig. 5B). This is the first demonstration of NEU1-mediated MUC1-ED desialylation and shedding in the bleomycin model, which forms the basis for future exploration of possible mechanistic contributions of shed MUC1-ED.

Effect of NEU1-Selective Inhibition with CG33300 in Acute Bleomycin Injury Model. To further support the benefit of therapeutic NEU1-selective inhibition in pulmonary fibrosis, we used a distinct NEU1-selective inhibitor: CG33300 (Guo et al., 2018). Similar to C9-BA-DANA (Fig. 3), CG33300 inhibited neuraminidase catalytic activity in vivo (Fig. 6A). In the acute bleomycin injury model, therapy with CG33300 that was started on day 7 after bleomycin challenge reversed the bleomycin-induced loss of body weight (Fig. 6B), attenuated bleomycin-provoked pulmonary lymphocytosis (Fig. 6C), and diminished accumulation of collagen in the lung tissue (Fig. 6D). These data indicate that two distinct NEU1selective inhibitors each attenuate the severity of bleomycininduced injury, thus expanding therapeutic options for NEU1 targeting in pulmonary fibrosis as well as mitigating the risks of inadvertent side effects of broad-spectrum neuraminidase inhibition.

\section{Discussion}

Early reports noted elevated sialidase activity in patients with diseases associated with fibrosis, including IPF, scleroderma, and sarcoidosis (Lambré et al., 1988; Komleva et al., 1995). We have recently reported that NEU1 is a central driver of pulmonary fibrosis, which acts by influencing diverse lung cell types (Luzina et al., 2016). Elevated NEU1 expression drives pulmonary inflammation and fibrosis in vivo and
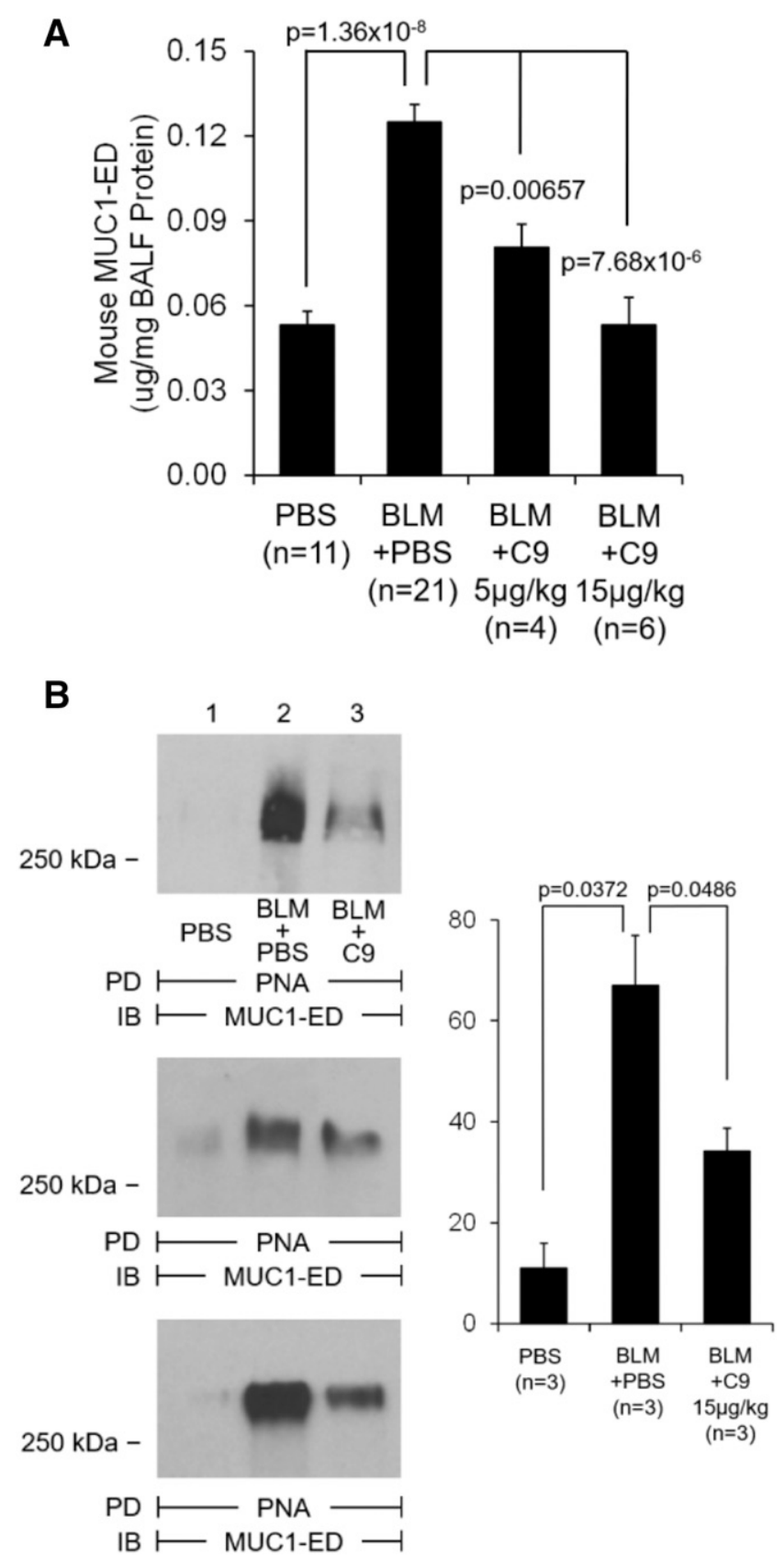

Fig. 5. NEU1-selective inhibition with C9-BA-DANA attenuates bleomycin-induced MUC1-ED shedding and desialylation. Mice were intratracheally challenged with bleomycin or PBS and then treated with C9-BA-DANA or with PBS as shown. Bronchoalveolar lavage fluids (BALFs) were collected on day 14 after bleomycin challenge (day 7 of treatment) and analyzed for total MUC1-ED by ELISA (A) and for desialylated MUC1-ED by PNA pull-down (PD) with subsequent Western immunoblotting (IB) for the MUC1-ED (B). In (B), mice from three independent experiments are shown, with the bar graph on the right showing mean \pm S.D. band densities.

introduces profibrotic cellular phenotypes in culture (Luzina et al., 2016). How these profibrotic activities are mediated is unknown. Others have extended our findings by demonstrating elevated expression of NEUs in the bleomycin model (Karhadkar et al., 2017) and suggested a profibrotic contribution from NEU3 (Karhadkar et al., 2020). Our findings of elevated NEU3 mRNA in patients with IPF (Fig. 2A) do not exclude such a contribution. In light of these existing reports, 

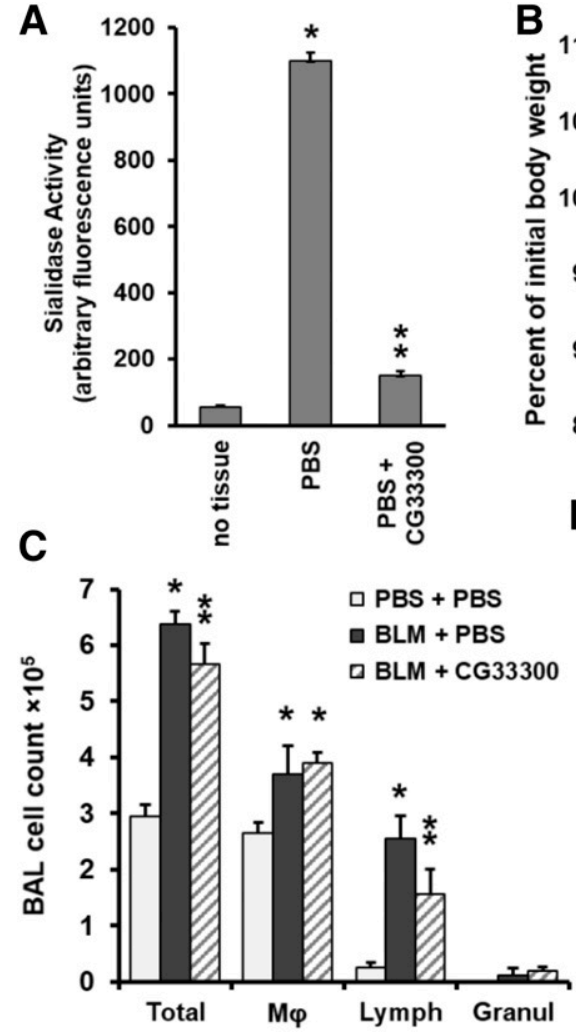

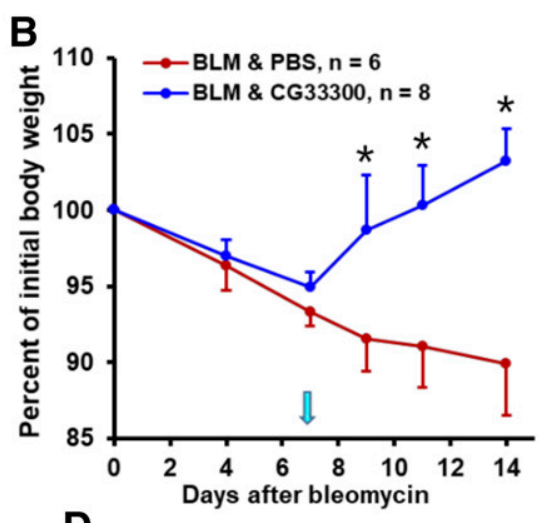

D

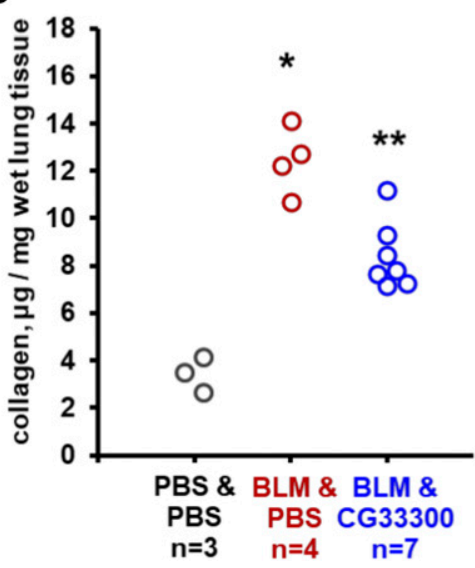

Fig. 6. NEU1-selective inhibition with CG33300 attenuates bleomycin-induced acute lung injury in mice. Mean \pm S.D. values are shown in (A-C). (A) Administration of CG33300 in vivo inhibits total neuraminidase activity, which is measured in arbitrary fluorescence units, in mouse lungs, four animals per group. Single asterisk denotes the basal total neuraminidase activity signal vs. no tissue $(P<$ $0.05)$, whereas double asterisk indicates a significant $(P<$ 0.05) CG33300-mediated decrease in total neuraminidase activity in the lung tissue. (B) Time-dependent changes in total body weight after intratracheal bleomycin challenge on day 0 followed by therapy with daily PBS (red, placebo) or CG33300 (blue) intraperitoneal injections starting on day 7 (arrow). Asterisks indicate significant $(P<0.05)$ recovery of body weight in response to CG33300 treatment. (C) Total and differential cell counts in bronchoalveolar lavage samples from mouse lungs on day 14 after bleomycin instillation followed by therapy with PBS or CG33300, three to seven animals per group. Single asterisks indicate significant $(P<0.05)$ increases in bleomycin-challenged animals compared with PBS controls, whereas double asterisks indicate significant $(P<0.05)$ decreases in CG33300-treated compared with PBS-treated bleomycinchallenged animals. (D) Collagen protein in microgram per milligram of wet lung tissue measured with the hydroxyproline assay (QuickZyme) on day 14 after the bleomycin challenge. Circles represent individual mice. Single asterisks indicate significant $(P<0.05)$ increases in bleomycinchallenged mice, and double asterisks indicate significant $(P<0.05)$ decreases induced by CG33300 therapy. it was not surprising to find that the pan-neuraminidase inhibitor DANA was protective in the acute bleomycin model in mice (Fig. 1).

We then tested which of the four neuraminidases was more likely to play a role in pulmonary fibrosis and observed that NEU1 was predominantly expressed. Furthermore, NEU1 was elevated in the lungs of human patients with IPF in their pulmonary fibroblasts as well as in the lungs of bleomycinchallenged mice (Fig. 2). The NEU1 chaperone and binding partner PPCA was also elevated at both mRNA and protein levels (Fig. 2). These findings raised the possibility that NEU1-selective inhibition may be therapeutic similar to pan-neuraminidase inhibition (Fig. 1). Two such NEU1selective inhibitors have been recently reported, including C9-BA-DANA (Magesh et al., 2008; Hyun et al., 2016; Lillehoj et al., 2019) and CG33300 (Guo et al., 2018; Howlader et al., 2020). Both compounds exploit modifications at the C9 position of the DANA scaffold based on structural selectivity-based hypotheses, but no related structural data for NEU1 are available. Indeed, the NEU1-selective inhibitor C9-BA-DANA was protective in both the acute and chronic bleomycin injury models in mice (Fig. 4). This inhibitor also attenuated MUC1ED desialylation and shedding (Fig. 5). A distinct NEU1selective inhibitor, CG33300, was similarly protective in the acute bleomycin injury model (Fig. 6).

Thus, NEU1-selective inhibition is as effective as broadspectrum inhibition of total neuraminidase activity for the protection against bleomycin-provoked pulmonary fibrosis. The more precise targeting of a single neuraminidase, NEU1, arguably minimizes the risk of potential adverse effects associated with nonselective inhibition of NEU2-4. In fact, no toxic effects of NEU1 inhibition with C9-BA-DANA or CG33300 were observed in this study with the doses tested within the experimental time frame. Inhibitors that target different NEU isoenzymes have shown disparate effects when tested in vitro (Howlader et al., 2020).

Our findings are translationally relevant, as they form the basis for the development of innovative therapies for pulmonary inflammation and fibrosis. However, the mechanisms through which NEU1 expression contributes to pulmonary fibrosis remain unclear. Our earlier findings suggested that higher NEU1 levels promote profibrotic phenotypes in multiple pulmonary cell types-epithelial, endothelial, stromal, immune-which are all known to contribute to fibrosis. On the molecular level, NEU1 may facilitate fibrosis by targeting sialic (neuraminic) acid residues that often occupy the outermost positions of protein glycan chains. The highly electronegative sialic acid influences protein tertiary conformation and in its terminal location is strategically positioned to influence intermolecular and cell-cell interactions through steric hindrance and/or electrostatic repulsion. Desialylation of a glycoprotein may mask or unmask cryptic binding sites, leading to functional consequences (Li and Chen, 2012; Miyagi and Yamaguchi, 2012). A number of sialoproteins that reportedly contribute to pathogenesis of interstitial lung disease are established NEU1 substrates, including epidermal growth factor receptor (Liu et al., 2011; Lillehoj et al., 2012); plateletderived growth factor receptor (Hinek et al., 2008); toll-like receptors 4, 7, and 9 (Meneghin et al., 2008; Amith et al., 2010; Trujillo et al., 2010; Abdulkhalek et al., 2011; Samara et al., 2012; Abdulkhalek and Szewczuk, 2013; Bhattacharyya et al., 2013); Fas/Fas ligand (Suzuki et al., 2003; Wynes et al., 2011; 
Wallach-Dayan et al., 2015); integrins $\alpha \mathrm{V} \beta 3$ and $\alpha \mathrm{V} \beta 5$ (Luzina et al., 2009; Munger and Sheppard, 2011; Chiodelli et al., 2012); CD31 (Lee et al., 2014a); and CD44 (Teder et al., 2002; Katoh et al., 2010; Li et al., 2011; Faller and Guvench, 2014) as well as the ligands for CD62L (Hamaguchi et al., 2002; Rosen, 2004). Additionally, increased NEU activity may mediate inflammation by distorting glycosphingolipid metabolism (Nowling et al., 2015, 2020). These previous observations implicate neuraminidases as important pathophysiological regulators of pulmonary inflammation and fibrosis.

Our findings presented in Fig. 5 suggest that NEU1 may mediate pulmonary fibrosis through a recently proposed but as yet not fully understood mechanism that is based on an airway mucin, MUC1. We discovered that MUC1 is a NEU1 substrate and that increased levels of soluble MUC1-ED, which is also referred to as KL-6, offer an indirect but specific measure of NEU1 catalytic activity (Lillehoj et al., 2015, 2019). KL-6 has also been extensively used as a biomarker for pulmonary fibrosis (Yokoyama et al., 2006; Wakamatsu et al., 2017; Ishii et al., 2018; Raghu et al., 2018). Therefore, elevated NEU1-mediated MUC1-ED desialylation and shedding may explain the commonly accepted association of fibrosis with elevated KL-6. We have expanded these findings further and consequently propose that elevated NEU1 acts as a facilitator of desialylation and shedding of MUC1, which then acts as a mechanistic profibrotic contributor (Xu et al., 2013; Ballester et al., 2019, 2020; Milara et al., 2020).

In summary, NEU1-selective inhibition offers antiinflammatory and antifibrotic therapeutic effects in the acute and chronic bleomycin models, likely by modulating multiple cellular and molecular mechanisms. Further development of NEU1-selective inhibitors has the potential to bring them to first-in-human trials and, ultimately, to approval as a new class of anti-inflammatory and antifibrotic therapies.

\section{Authorship Contributions}

Participated in research design: Luzina, Cairo, Atamas, Goldblum. Conducted experiments: Luzina, Lillehoj, Lockatell, Hyun, Lugkey, Imamura, Ishida, Cairo.

Contributed new reagents or analytic tools: Imamura, Ishida, Cairo.

Performed data analysis: Luzina, Atamas, Goldblum.

Wrote or contributed to the writing of the manuscript: Luzina, Atamas, Goldblum.

\section{References}

Abdulkhalek S, Amith SR, Franchuk SL, Jayanth P, Guo M, Finlay T, Gilmour A, Guzzo C, Gee K, Beyaert R, et al. (2011) Neu1 sialidase and matrix metalloproteinase-9 cross-talk is essential for Toll-like receptor activation and cellular signaling. J Biol Chem 286:36532-36549.

Abdulkhalek S and Szewczuk MR (2013) Neu1 sialidase and matrix metalloproteinase-9 cross-talk regulates nucleic acid-induced endosomal TOLL like receptor-7 and -9 activation, cellular signaling and pro-inflammatory responses. Cell Signal 25:2093-2105.

Amith SR, Jayanth P, Franchuk S, Finlay T, Seyrantepe V, Beyaert R, Pshezhetsky AV, and Szewczuk MR (2010) Neu1 desialylation of sialyl alpha-2,3-linked betagalactosyl residues of TOLL-like receptor 4 is essential for receptor activation and cellular signaling. Cell Signal 22:314-324.

Ballester B, Milara J, and Cortijo J (2019) Mucins as a new frontier in pulmonary fibrosis. J Clin Med 8:1447.

Ballester B, Milara J, and Cortijo J (2020) Pirfenidone anti-fibrotic effects are partially mediated by the inhibition of MUC1 bioactivation. Oncotarget 11:1306-1320. Baran CP, Opalek JM, McMaken S, Newland CA, O'Brien JM Jr, Hunter MG, Bringardner BD, Monick MM, Brigstock DR, Stromberg PC, et al. (2007) Importan roles for macrophage colony-stimulating factor, $\mathrm{CC}$ chemokine ligand 2, and mononuclear phagocytes in the pathogenesis of pulmonary fibrosis. Am J Respir Crit Care Med 176:78-89.

Bhattacharyya S, Kelley K, Melichian DS, Tamaki Z, Fang F, Su Y, Feng G, Pope RM, Budinger GR, Mutlu GM, et al. (2013) Toll-like receptor 4 signaling augments transforming growth factor- $\beta$ responses: a novel mechanism for maintaining and amplifying fibrosis in scleroderma. Am J Pathol 182:192-205.
Carrington R, Jordan S, Pitchford SC, and Page CP (2018) Use of animal models in IPF research. Pulm Pharmacol Ther 51:73-78.

Chairat K, Tarning J, White NJ, and Lindegardh N (2013) Pharmacokinetic properties of anti-influenza neuraminidase inhibitors. J Clin Pharmacol 53:119-139. Chiodelli P, Urbinati C, Mitola S, Tanghetti E, and Rusnati M (2012) Sialic acid associated with $\alpha \mathrm{v} \beta 3$ integrin mediates HIV-1 Tat protein interaction and endothelial cell proangiogenic activation. $J$ Biol Chem 287:20456-20466.

Cross AS, Hyun SW, Miranda-Ribera A, Feng C, Liu A, Nguyen C, Zhang L, Luzina IG, Atamas SP, Twaddell WS, et al. (2012) NEU1 and NEU3 sialidase activity expressed in human lung microvascular endothelia: NEU1 restrains endothelial cell migration, whereas NEU3 does not. J Biol Chem 287:15966-15980.

Degryse AL and Lawson WE (2011) Progress toward improving animal models for idiopathic pulmonary fibrosis. Am J Med Sci 341:444-449.

Duca L, Blanchevoye C, Cantarelli B, Ghoneim C, Dedieu S, Delacoux F, Hornebeck W, Hinek A, Martiny L, and Debelle L (2007) The elastin receptor complex transduces signals through the catalytic activity of its Neu-1 subunit. J Biol Chem 282:12484-12491.

Faller CE and Guvench O (2014) Terminal sialic acids on CD44 N-glycans can block hyaluronan binding by forming competing intramolecular contacts with arginine sidechains. Proteins 82:3079-3089.

Fischer A and Distler J (2019) Progressive fibrosing interstitial lung disease associated with systemic autoimmune diseases. Clin Rheumatol 38:2673-2681.

George PM, Wells AU, and Jenkins RG (2020) Pulmonary fibrosis and COVID-19: the potential role for antifibrotic therapy. Lancet Respir Med 8:807-815.

Glasser SW, Hagood JS, Wong S, Taype CA, Madala SK, and Hardie WD (2016) Mechanisms of lung fibrosis resolution. Am J Pathol 186:1066-1077.

Greenberger PA (2019) Hypersensitivity pneumonitis: A fibrosing alveolitis produced by inhalation of diverse antigens. $J$ Allergy Clin Immunol 143:1295-1301.

Guo T, Héon-Roberts R, Zou C, Zheng R, Pshezhetsky AV, and Cairo CW (2018) Selective inhibitors of human neuraminidase 1 (NEU1). J Med Chem 61: $11261-11279$

Gupta YK, Meenu M, and Mohan P (2015) The Tamiflu fiasco and lessons learnt. Indian J Pharmacol 47:11-16.

Hamaguchi Y, Nishizawa Y, Yasui M, Hasegawa M, Kaburagi Y, Komura K, Nagaoka T, Saito E, Shimada Y, Takehara K, et al. (2002) Intercellular adhesion molecule-1 and L-selectin regulate bleomycin-induced lung fibrosis. Am J Pathol 161:1607-1618. Hanania AN, Mainwaring W, Ghebre YT, Hanania NA, and Ludwig M (2019) Radiation-induced lung injury: assessment and management. Chest 156:150-162

Hata K, Koseki K, Yamaguchi K, Moriya S, Suzuki Y, Yingsakmongkon S, Hirai G, Sodeoka M, von Itzstein M, and Miyagi T (2008) Limited inhibitory effects of oseltamivir and zanamivir on human sialidases. Antimicrob Agents Chemother $\mathbf{5 2}$ : 3484-3491.

Hattrup CL and Gendler SJ (2008) Structure and function of the cell surface (tethered) mucins. Annu Rev Physiol 70:431-457.

Heneghan CJ, Onakpoya I, Jones MA, Doshi P, Del Mar CB, Hama R, Thompson MJ, Spencer EA, Mahtani KR, Nunan D, et al. (2016) Neuraminidase inhibitors for influenza: a systematic review and meta-analysis of regulatory and mortality data. Health Technol Assess 20:1-242.

Hinek A, Bodnaruk TD, Bunda S, Wang Y, and Liu K (2008) Neuraminidase-1, a subunit of the cell surface elastin receptor, desialylates and functionally inactivates adjacent receptors interacting with the mitogenic growth factors PDGF-BB and IGF-2. Am J Pathol 173:1042-1056.

Hinek A, Pshezhetsky AV, von Itzstein M, and Starcher B (2006) Lysosomal sialidase (neuraminidase-1) is targeted to the cell surface in a multiprotein complex that facilitates elastic fiber assembly. $J$ Biol Chem 281:3698-3710.

Howlader MA, Guo T, Chakraberty R, and Cairo CW (2020) Isoenzyme-selective inhibitors of human neuraminidases reveal distinct effects on cell migration. ACS Chem Biol 15:1328-1339.

Hoy RF and Chambers DC (2020) Silica-related diseases in the modern world. Allergy DOI: $10.1111 /$ all.14202 [published ahead of print]

Hyun SW, Liu A, Liu Z, Cross AS, Verceles AC, Magesh S, Kommagalla Y, Kona C, Ando H, Luzina IG, et al. (2016) The NEU1-selective sialidase inhibitor, C9-butylamide-DANA, blocks sialidase activity and NEU1-mediated bioactivities in human lung in vitro and murine lung in vivo. Glycobiology 26:834-849.

Ishii H, Kushima H, Kinoshita Y, Fujita M, and Watanabe K (2018) The serum KL-6 levels in untreated idiopathic pulmonary fibrosis can naturally decline in association with disease progression. Clin Respir J 12:2411-2418.

Joseph R, D'Andrea G, Oster SB, and Welch KM (1989) Whole blood platelet function in acute ischemic stroke. Importance of dense body secretion and effects of antithrombotic agents. Stroke 20:38-44.

Karhadkar TR, Chen W, and Gomer RH (2020) Attenuated pulmonary fibrosis in sialidase-3 knockout $\left(\mathrm{Neu3}^{\left.-{ }^{-}\right)}\right.$) mice. Am J Physiol Lung Cell Mol Physiol 318: L165-L179.

Karhadkar TR, Pilling D, Cox N, and Gomer RH (2017) Sialidase inhibitors attenuate pulmonary fibrosis in a mouse model. Sci Rep 7:15069.

Katoh S, Maeda S, Fukuoka H, Wada T, Moriya S, Mori A, Yamaguchi K, Senda S, and Miyagi T (2010) A crucial role of sialidase Neu1 in hyaluronan receptor function of CD44 in Thelper type 2-mediated airway inflammation of murine acute asthmatic model. Clin Exp Immunol 161:233-241.

Khazdair MR, Boskabady MH, and Ghorani V (2015) Respiratory effects of sulfur mustard exposure, similarities and differences with asthma and COPD. Inhal Toxicol 27:731-744

Kolahian S, Fernandez IE, Eickelberg O, and Hartl D (2016) Immune mechanisms in pulmonary fibrosis. Am J Respir Cell Mol Biol 55:309-322.

Komleva AV, Prokazova NV, Gabrielyan ND, Mukhin DN, Kashnikova LN, Zvezdina ND, Martinova LE, and Panasyuk AF (1995) Phospholipids and glycosphingolipids in cultured skin fibroblasts from patients with progressive systemic sclerosis (scleroderma). Clin Exp Rheumatol 13:581-588.

Kurundkar A and Thannickal VJ (2016) Redox mechanisms in age-related lung fibrosis. Redox Biol 9:67-76. 
Lambré CR, Pilatte Y, Le Maho S, Greffard A, De Crémoux H, and Bignon J (1988) Sialidase activity and antibodies to sialidase-treated autologous erythrocytes in bronchoalveolar lavages from patients with idiopathic pulmonary fibrosis or sarcoidosis. Clin Exp Immunol 73:230-235.

Lederer DJ and Martinez FJ (2018) Idiopathic pulmonary fibrosis. N Engl J Med 378: 1811-1823.

Lee C, Liu A, Miranda-Ribera A, Hyun SW, Lillehoj EP, Cross AS, Passaniti A, Grimm PR, Kim BY, Welling PA, et al. (2014a) NEU1 sialidase regulates the sialylation state of CD31 and disrupts CD31-driven capillary-like tube formation in human lung microvascular endothelia. J Biol Chem 289:9121-9135.

Lee R, Reese C, Bonner M, Tourkina E, Hajdu Z, Riemer EC, Silver RM, Visconti RP, and Hoffman S (2014b) Bleomycin delivery by osmotic minipump: similarity to human scleroderma interstitial lung disease. Am J Physiol Lung Cell Mol Physiol 306:L736-L748.

Li Y and Chen X (2012) Sialic acid metabolism and sialyltransferases: natural functions and applications. Appl Microbiol Biotechnol 94:887-905.

Li Y, Jiang D, Liang J, Meltzer EB, Gray A, Miura R, Wogensen L, Yamaguchi Y, and Noble PW (2011) Severe lung fibrosis requires an invasive fibroblast phenotype regulated by hyaluronan and CD44. J Exp Med 208:1459-1471.

Lillehoj EP, Guang W, Hyun SW, Liu A, Hegerle N, Simon R, Cross AS, Ishida H, Luzina IG, Atamas SP, et al. (2019) Neuraminidase 1-mediated desialylation of the mucin 1 ectodomain releases a decoy receptor that protects against Pseudomonas aeruginosa lung infection. J Biol Chem 294:662-678.

Lillehoj EP, Hyun SW, Feng C, Zhang L, Liu A, Guang W, Nguyen C, Luzina IG, Atamas SP, Passaniti A, et al. (2012) NEU1 sialidase expressed in human airway epithelia regulates epidermal growth factor receptor (EGFR) and MUC1 protein signaling. J Biol Chem 287:8214-8231.

Lillehoj EP, Hyun SW, Liu A, Guang W, Verceles AC, Luzina IG, Atamas SP, Kim KC, and Goldblum SE (2015) NEU1 sialidase regulates membrane-tethered mucin (MUC1) ectodomain adhesiveness for Pseudomonas aeruginosa and decoy receptor release. J Biol Chem 290:18316-18331.

Lillehoj EP, Kato K, Lu W, and Kim KC (2013) Cellular and molecular biology of airway mucins. Int Rev Cell Mol Biol 303:139-202.

Lindemann L, Jacobsen H, Schuhbauer D, Knoflach F, Gatti S, Wettstein JG, Loetscher H, Chu T, Ebeling M, Paulson JC, et al. (2010) In vitro pharmacological selectivity profile of oseltamivir prodrug (Tamiflu) and active metabolite. Eur $J$ Pharmacol 628:6-10.

Liu YC, Yen HY, Chen CY, Chen CH, Cheng PF, Juan YH, Chen CH, Khoo KH, Yu CJ, Yang PC, et al. (2011) Sialylation and fucosylation of epidermal growth factor receptor suppress its dimerization and activation in lung cancer cells. Proc Nat Acad Sci USA 108:11332-11337.

Luo F, Le NB, Mills T, Chen NY, Karmouty-Quintana H, Molina JG, Davies J, Philip K, Volcik KA, Liu H, et al. (2016) Extracellular adenosine levels are associated with the progression and exacerbation of pulmonary fibrosis. FASEB $J$ 30:874-883.

Luzina IG, Kopach P, Lockatell V, Kang PH, Nagarsekar A, Burke AP, Hasday JD, Todd NW, and Atamas SP (2013) Interleukin-33 potentiates bleomycin-induced lung injury. Am J Respir Cell Mol Biol 49:999-1008.

Luzina IG, Lockatell V, Hyun SW, Kopach P, Kang PH, Noor Z, Liu A, Lillehoj EP, Lee C, Miranda-Ribera A, et al. (2016) Elevated expression of NEU1 sialidase in idiopathic pulmonary fibrosis provokes pulmonary collagen deposition, lymphocytosis, and fibrosis. Am J Physiol Lung Cell Mol Physiol 310:L940-L954.

Luzina IG, Lockatell V, Todd NW, Kopach P, Pentikis HS, and Atamas SP (2015a) Pharmacological in vivo inhibition of S-nitrosoglutathione reductase attenuates bleomycin-induced inflammation and fibrosis. J Pharmacol Exp Ther 355:13-22.

Luzina IG, Todd NW, Nacu N, Lockatell V, Choi J, Hummers LK, and Atamas SP (2009) Regulation of pulmonary inflammation and fibrosis through expression of integrins alphaVbeta3 and alphaVbeta5 on pulmonary T lymphocytes. Arthritis Rheum 60:1530-1539.

Luzina IG, Todd NW, Sundararajan S, and Atamas SP (2015b) The cytokines of pulmonary fibrosis: much learned, much more to learn. Cytokine 74:88-100.

Magesh S, Moriya S, Suzuki T, Miyagi T, Ishida H, and Kiso M (2008) Design, synthesis, and biological evaluation of human sialidase inhibitors. Part 1: selective inhibitors of lysosomal sialidase (NEU1). Bioorg Med Chem Lett 18:532-537.

Meneghin A, Choi ES, Evanoff HL, Kunkel SL, Martinez FJ, Flaherty KR, Toews GB, and Hogaboam CM (2008) TLR9 is expressed in idiopathic interstitial pneumonia and its activation promotes in vitro myofibroblast differentiation. Histochem Cell Biol 130:979-992.

Milara J, Ballester B, Montero P, Escriva J, Artigues E, Alós M, Pastor-Clerigues A, Morcillo E, and Cortijo J (2020) MUC1 intracellular bioactivation mediates lung fibrosis. Thorax 75:132-142.

Miyagi T and Yamaguchi K (2012) Mammalian sialidases: physiological and pathological roles in cellular functions. Glycobiology 22:880-896.

Moore BB, Lawson WE, Oury TD, Sisson TH, Raghavendran K, and Hogaboam CM (2013) Animal models of fibrotic lung disease. Am J Respir Cell Mol Biol 49: 167-179.

Munger JS and Sheppard D (2011) Cross talk among TGF- $\beta$ signaling pathways, integrins, and the extracellular matrix. Cold Spring Harb Perspect Biol 3:a005017.

Naik PK and Moore BB (2010) Viral infection and aging as cofactors for the development of pulmonary fibrosis. Expert Rev Respir Med 4:759-771.

Nowling TK, Mather AR, Thiyagarajan T, Hernández-Corbacho MJ, Powers TW, Jones EE, Snider AJ, Oates JC, Drake RR, and Siskind LJ (2015) Renal glycosphingolipid metabolism is dysfunctional in lupus nephritis. J Am Soc Nephrol 26 1402-1413.
Nowling TK, Rodgers J, Thiyagarajan T, Wolf B, Bruner E, Sundararaj K, Molano I, and Gilkeson G (2020) Targeting glycosphingolipid metabolism as a potential therapeutic approach for treating disease in female MRL/lpr lupus mice. PLoS One 15:e0230499.

O'Dwyer DN and Moore BB (2018) Animal models of pulmonary fibrosis. Methods Mol Biol 1809:363-378.

Perelas A, Silver RM, Arrossi AV, and Highland KB (2020) Systemic sclerosisassociated interstitial lung disease. Lancet Respir Med 8:304-320.

Pochetuhen K, Luzina IG, Lockatell V, Choi J, Todd NW, and Atamas SP (2007) Complex regulation of pulmonary inflammation and fibrosis by CCL18. Am $J$ Pathol 171:428-437.

Raghu G, Richeldi L, Jagerschmidt A, Martin V, Subramaniam A, Ozoux ML, Esperet CA, and Soubrane C (2018) Idiopathic pulmonary fibrosis: prospective, case-controlled study of natural history and circulating biomarkers. Chest 154: 1359-1370.

Ravanetti F, Ragionieri L, Ciccimarra R, Ruscitti F, Pompilio D, Gazza F, Villetti G, Cacchioli A, and Stellari FF (2020) Modeling pulmonary fibrosis through bleomycin delivered by osmotic minipump: a new histomorphometric method of evaluation. Am J Physiol Lung Cell Mol Physiol 318:L376-L385.

Richards MR, Guo T, Hunter CD, and Cairo CW (2018) Molecular dynamics simulations of viral neuraminidase inhibitors with the human neuraminidase enzymes: insights into isoenzyme selectivity. Bioorg Med Chem 26:5349-5358.

Rosen SD (2004) Ligands for L-selectin: homing, inflammation, and beyond. Annu Rev Immunol 22:129-156.

Samara KD, Antoniou KM, Karagiannis K, Margaritopoulos G, Lasithiotaki I, Koutala E, and Siafakas NM (2012) Expression profiles of Toll-like receptors in nonsmall cell lung cancer and idiopathic pulmonary fibrosis. Int J Oncol 40:1397-1404.

Spagnolo P, Lee JS, Sverzellati N, Rossi G, and Cottin V (2018) The lung in rheumatoid arthritis: focus on interstitial lung disease. Arthritis Rheumatol 70: $1544-1554$

Storr SJ, Royle L, Chapman CJ, Hamid UM, Robertson JF, Murray A, Dwek RA, and Rudd PM (2008) The O-linked glycosylation of secretory/shed MUC1 from an advanced breast cancer patient's serum. Glycobiology 18:456-462.

Suzuki O, Nozawa Y, and Abe M (2003) Sialic acids linked to glycoconjugates of Fas regulate the caspase-9-dependent and mitochondria-mediated pathway of Fasinduced apoptosis in Jurkat T cell lymphoma. Int J Oncol 23:769-774.

Teder P, Vandivier RW, Jiang D, Liang J, Cohn L, Puré E, Henson PM, and Noble PW (2002) Resolution of lung inflammation by CD44. Science 296:155-158.

Todd NW, Luzina IG, and Atamas SP (2012) Molecular and cellular mechanisms of pulmonary fibrosis. Fibrogenesis Tissue Repair 5:11.

Trethewey SP and Walters GI (2018) The role of occupational and environmental exposures in the pathogenesis of idiopathic pulmonary fibrosis: a narrative literature review. Medicina (Kaunas) 54:108.

Trujillo G, Meneghin A, Flaherty KR, Sholl LM, Myers JL, Kazerooni EA, Gross BH, Oak SR, Coelho AL, Evanoff H, et al. (2010) TLR9 differentiates rapidly from slowly progressing forms of idiopathic pulmonary fibrosis. Sci Transl Med 2: $57 \mathrm{ra} 82$

Varone F, Iovene B, Sgalla G, Calvello M, Calabrese A, Larici AR, and Richeldi L (2020) Fibrotic hypersensitivity pneumonitis: diagnosis and management. Lung 198:429-440.

Wakamatsu K, Nagata N, Kumazoe H, Oda K, Ishimoto H, Yoshimi M, Takata S, Hamada M, Koreeda Y, Takakura K, et al. (2017) Prognostic value of serial serum KL-6 measurements in patients with idiopathic pulmonary fibrosis. Respir Investig 55:16-23.

Wallach-Dayan SB, Elkayam L, Golan-Gerstl R, Konikov J, Zisman P, Dayan MR, Arish N, and Breuer R (2015) Cutting edge: FasL(+) immune cells promote resolution of fibrosis. $J$ Autoimmun 59:67-76.

Weng T, Ko J, Masamha CP, Xia Z, Xiang Y, Chen NY, Molina JG, Collum S, Mertens TC, Luo F, et al. (2019) Cleavage factor 25 deregulation contributes to pulmonary fibrosis through alternative polyadenylation. J Clin Invest 129:1984-1999.

Wynes MW, Edelman BL, Kostyk AG, Edwards MG, Coldren C, Groshong SD, Cosgrove GP, Redente EF, Bamberg A, Brown KK, et al. (2011) Increased cell surface Fas expression is necessary and sufficient to sensitize lung fibroblasts to Fas ligation-induced apoptosis: implications for fibroblast accumulation in idiopathic pulmonary fibrosis. J Immunol 187:527-537.

Wynn TA (2011) Integrating mechanisms of pulmonary fibrosis. $J$ Exp Med 208: 1339-1350.

Xu L, Yang D, Zhu S, Gu J, Ding F, Bian W, Rong Z, and Shen C (2013) Bleomycininduced pulmonary fibrosis is attenuated by an antibody against KL-6. Exp Lung Res 39:241-248.

Yokoyama A, Kondo K, Nakajima M, Matsushima T, Takahashi T, Nishimura M, Bando M, Sugiyama Y, Totani Y, Ishizaki T, et al. (2006) Prognostic value of circulating KL-6 in idiopathic pulmonary fibrosis. Respirology 11:164-168.

Zhou Y, Schneider DJ, Morschl E, Song L, Pedroza M, Karmouty-Quintana H, Le T, Sun CX, and Blackburn MR (2011) Distinct roles for the A2B adenosine receptor in acute and chronic stages of bleomycin-induced lung injury. J Immunol 186: 1097-1106.

Address correspondence to: Dr. Irina G. Luzina, Department of medicine, University of Maryland School of Medicine, 10 S. Pine St., MSTF 8-34, Baltimore, MD 21201. E-mail: iluzina@som.umaryland.edu 